\title{
Journal of Bone and Mineral Metabolism Best Paper Award 2012
}

\author{
(c) The Japanese Society for Bone and Mineral Research and Springer Japan 2013
}

The Journal of Bone and Mineral Metabolism Best Paper Award was established in 2008. Candidates for the award must be members of the Japanese Society of Bone and Mineral Research, and the winner is honored at the Society's Annual Meeting.

We are pleased to announce that the following article has received JBMM Best Paper Award.

"Prevalence of knee osteoarthritis, lumbar spondylosis, and osteoporosis in Japanese men and women: the research on osteoarthritis/osteoporosis against disability study" by

Noriko Yoshimura, Shigeyuki Muraki, Hiroyuki Oka, Akihiko Mabuchi, Yoshio En-Yo, Munehito Yoshida, Akihiko Saika, Hideyo Yoshida, Takao Suzuki, Seizo Yamamoto, Hideaki Ishibashi, Hiroshi Kawaguchi, Kozo Nakamura, Toru Akune

J Bone Miner Metab (2009) 27:620-628

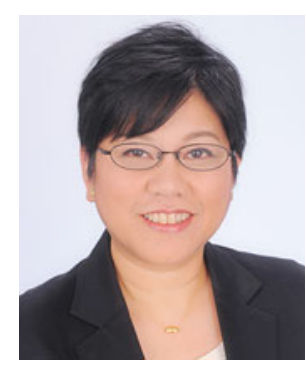

Dr. Noriko Yoshimura

\begin{abstract}
Musculoskeletal diseases, especially osteoarthritis (OA) and osteoporosis (OP), impair activities of daily life (ADL) and quality of life (QOL) in the elderly. Although preventive strategies for these diseases are urgently required in an aging society, epidemiological data on these diseases are scant. To clarify the prevalence of knee osteoarthritis (KOA), lumbar spondylosis (LS), and osteoporosis (OP) in Japan, and estimate the number of people with these diseases, we started a large-scale population-based cohort study entitled research on osteoarthritis/osteoporosis against disability (ROAD) in 2005. This study involved the collection of clinical information from three cohorts composed of participants located in urban, mountainous, and coastal areas. KOA and LS were radiographically defined as a grade of $\geq 2$ by the Kellgren-Lawrence scale; OP was defined by the criteria of the Japanese Society for Bone and Mineral Research. The 3,040 participants in total were divided into six groups based on their age: $\leq 39,40-49,50-59,60-69,70-79$, and $\geq 80$ years. The prevalence of KOA in the age groups $\leq 39,40-49,50-59,60-69,70-79$, and $\geq 80$ years $0,9.1,24.3$, $35.2,48.2$, and $51.6 \%$, respectively, in men, and the prevalence in women of the same age groups was $3.2,11.4,30.3,57.1,71.9$, and $80.7 \%$, respectively. With respect to the age groups, the prevalence of LS was $14.3,45.5,72.9,74.6,85.3$, and 90.1 \% in men, and 9.7, 28.6, 41.7, 55.4, 75.1, and 78.2\% in women, respectively. Data of the prevalence of OP at the lumbar spine and femoral neck were also obtained. The estimated number of patients with KOA, LS, and L2-L4 and femoral neck OP in Japan was approximately $25,38,6.4$, and 11 million, respectively. In summary, we estimated the prevalence of OA and OP, and the number of people affected with these diseases in Japan. The ROAD study will elucidate epidemiological evidence concerning determinants of bone and joint disease.
\end{abstract}

We offer our sincere congratulations on behalf of the Journal of Bone and Mineral Metabolism, with best wishes for further development of the author's research.

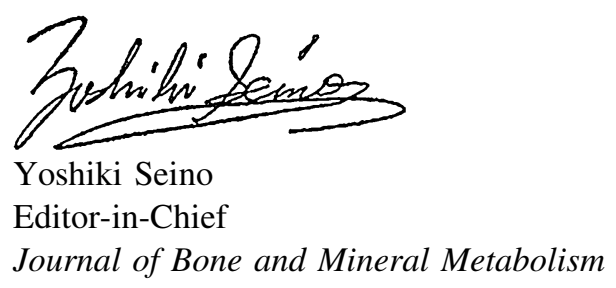

